OPEN ACCESS

Edited by: Otto Metzger, Dana-Farber Cancer Institute, United States

Reviewed by: Tomas Reinert, Universidade Federal do Rio Grande do Sul (UFRGS), Brazil

Carmine De Angelis, Baylor College of Medicine, United States

*Correspondence: Tarah J. Ballinger tarahb@iu.edu

Specialty section

This article was submitted to

Women's Cancer

a section of the journal

Frontiers in Oncology

Received: 13 March 2018

Accepted: 20 July 2018

Published: 10 August 2018

Citation:

Ballinger TJ, Meier JB and Jansen VM (2018) Current Landscape of Targeted

Therapies for Hormone-Receptor Positive, HER2 Negative Metastatic

Breast Cancer. Front. Oncol. 8:308. doi: 10.3389/fonc. 2018.00308

\section{Current Landscape of Targeted Therapies for Hormone-Receptor Positive, HER2 Negative Metastatic Breast Cancer}

\author{
Tarah J. Ballinger ${ }^{1 *}$, Jason B. Meier ${ }^{2}$ and Valerie M. Jansen ${ }^{2}$ \\ ${ }^{1}$ Division of Hematology-Oncology, Department of Medicine, Indiana University School of Medicine, Indianapolis, IN, \\ United States, ${ }^{2}$ Division of Hematology-Oncology, Department of Medicine, Vanderbilt University Medical Center, Nashville, \\ TN, United States
}

The majority of deaths from MBC are in patients with hormone receptor (HR) positive, HER2 negative disease. Endocrine therapy (ET) remains the backbone of treatment in these cases, improving survival and quality of life. However, treatment can lose effectiveness due to primary or acquired endocrine resistance. Analysis of mechanisms of ET resistance has led to the development of a new generation of targeted therapies for advanced breast cancer. In addition to anti-estrogen therapy with selective estrogen receptor modulators, aromatase inhibitors, and/or selective estrogen receptor degraders, combinations with cyclin dependent kinase (CDK) 4/6 inhibitors have led to substantial progression free survival (PFS) improvements in the first and second line settings. While the PI3K/AKT/mTOR pathway is known to be an important growth pathway in HR positive breast cancer, PI3K inhibitors have been disappointing due to modest effect sizes and significant toxicity. The mTOR inhibitor everolimus significantly improves progression free survival when added to ET, and recent studies have improved supportive care allowing less toxicity. While these combination targeted therapies improve outcomes and often delay initiation of chemotherapy, long term overall survival data are lacking and data for the ideal strategy for sequencing these agents remains unclear. Ongoing research evaluating potential biomarkers and mechanisms of resistance is anticipated to continue to improve outcomes for patients with HR positive metastatic breast cancer. In this review, we will discuss management and ongoing challenges in the treatment of advanced HR positive, HER2 negative breast cancer, highlighting single agent and combination endocrine therapies, targeted therapies including palbociclib, ribociclib, abemaciclib, and everolimus, and sequencing of therapies in the clinic.

Keywords: breast cancer, endocrine therapy, cyclin dependent kinase inhibitor, endocrine resistance, targeted therapy

\section{INTRODUCTION}

With approximately 250,000 new cases and 40,000 deaths annually, breast cancer is the most common malignancy and a leading cause of cancer-related death for women in the United States (1). The majority of breast cancers are hormone receptor positive (HR positive), including estrogen receptor (ER) and/or progesterone receptor (PR) positive, with endocrine therapy (ET) remaining 
the backbone of therapy (2-4). Approximately 5-10\% of breast cancer patients present with de novo metastatic disease, and $30 \%$ of those initially treated with ET for early stage disease will relapse with metastatic spread (2). In the setting of metastatic breast cancer $(\mathrm{MBC})$, the goal of treatment is palliative, and ET plays a crucial role of maintaining disease control, delaying the need for chemotherapy, and preserving quality of life. Multiple new agents have been developed to treat patients in the event of endocrine resistance; however, optimal sequencing of these therapies has not yet been determined in clinical trials. Herein, we present a review of the current treatment landscape for optimal management of HR positive, HER2 negative MBC.

\section{ENDOCRINE THERAPIES}

ET includes the selective estrogen receptor modulators (SERMs; tamoxifen), aromatase inhibitors (AIs; letrozole, anastrozole, exemestane) and selective estrogen receptor down regulators (SERDs; fulvestrant). Given the heterogeneity of disease presentation and patient characteristics, there is no standard consensus recommendation for the optimal sequencing of these agents in patients with HR positive breast cancer in the metastatic setting (5-8). Results from several phase III studies evaluating single agent and combination endocrine therapy are detailed in Table 1.

One of the oldest of these therapies is the SERM tamoxifen, approved by the FDA as adjuvant therapy in 1986 and repeatedly found to be effective in the metastatic setting. A systematic review of 86 clinical trials showed an overall response rate (ORR) of $34 \%$ for single agent tamoxifen (9). In pre-menopausal patients with $\mathrm{MBC}$, first-line therapy with ovarian ablation with either goserelin or buserelin and tamoxifen was associated with a significant increase in OS when compared to ovarian ablation alone (HR 0.78, 95\% CI 0.63-0.96, $p=0.02$ ), with a significant improvement in PFS as well (HR 0.70, 95\% CI 0.59-0.85) (10). For post-menopausal patients, in whom estrogen synthesis occurs in the peripheral tissues through aromatization, treatment with AIs has largely replaced tamoxifen. There is no clinical evidence to suggest favoring one AI over another. Although there are mechanistic differences between the steroidal AI (exemestane) and non-steroidal AIs (letrozole and anastrozole) (11), they are not fully cross-resistant (12). AIs have been associated with improved overall response rate (ORR), time to progression (TTP) and disease control rate when compared to tamoxifen (13). A large meta-analysis demonstrated superior survival for patients receiving AI compared to tamoxifen (HR 0.89 , 95\% CI 0.80-0.99) (9). Side effect profiles of AIs and SERMs vary, with AIs increasing rates of bone loss and musculoskeletal side effects, and tamoxifen carrying an increased risk of thromboembolic disease and uterine cancer (14).

Treatment with SERDs represents another viable option for ET. Initial studies of fulvestrant $250 \mathrm{mg}$ showed modest antitumor activity, while higher dosing with $500 \mathrm{mg}$ has been found to provide superior disease control and a greater survival advantage in comparison to AIs (15-18). In the FIRST study of 205 post-menopausal patients with advanced breast cancer, fulvestrant $500 \mathrm{mg}$ was found to have a superior TTP and a decreased risk of progression when compared to anastrozole (19). Subsequent analysis of the FALCON trial comparing fulvestrant $500 \mathrm{mg}$ and anastrozole in the first-line metastatic setting found that fulvestrant was associated with a significant improvement in PFS (16.6 months vs. 13.8 months, $p=0.048$ ), with similar rates of adverse events such as arthralgias and hot flashes (20). Of note, most of the patients in the FALCON study had never received prior endocrine therapy. While fulvestrant alone remains an option for endocrine naïve patients in the first line setting, single agent response rates are low in previously treated patients (21) and the majority of patients will now receive a CDK4/6 inhibitor, as discussed later in this review.

\section{COMBINATION ENDOCRINE THERAPY}

Combination therapy with anti-endocrine agents is a logical extension of single agent anti-endocrine therapy, given the mechanistic differences between the agents. Based on encouraging preclinical studies, combination treatment with fulvestrant plus anastrozole was compared to single agent anastrozole in the FACT and SWOG S0226 trials. Of note, both trials used a lower fulvestrant dosing schedule (500 mg loading, then 250 mg monthly). In the FACT trial, combination endocrine therapy did not demonstrate any significant improvement in TTP or OS (22). In the SWOG S0226 trial, combination therapy was associated with an improved PFS, [13.5 months vs. 15.0 months (HR $0.895 \%$ CI $0.68-0.94 ; p=0.007$ )] and OS [41.3 months vs. 47.7 months (HR $0.8195 \%$ CI $0.65-1.00$; $p=0.05)]$ (23). The difference in these results likely has to do with differing populations in each study. While the SWOG S0226 trial participants were largely treatment naive, approximately two thirds of participants in the FACT trial had received previous anti-estrogen therapy.

Given the lack of benefit for combination anti-endocrine therapy seen in the FACT trial, the South Korean SoFEA trial was explicitly designed to address the population of $\mathrm{MBC}$ patients with progression on prior non-steroidal AI therapy. In a randomized three arm trial, combination therapy with fulvestrant (500 mg loading dose and $250 \mathrm{mg}$ monthly) plus anastrozole was compared to fulvestrant plus placebo or single agent exemestane. No significant differences were identified between the combination arm and either of the single agent arms for PFS or OS (24). A subsequent Cochrane Review of fulvestrant in $\mathrm{MBC}$ incorporating 4,514 patients found no benefit for fulvestrant when used in combination ET in the first or second line settings (25).

\section{MECHANISMS OF ENDOCRINE RESISTANCE}

A major obstacle in the treatment of $\mathrm{HR}$ positive $\mathrm{MBC}$ is intrinsic or acquired resistance to ET. There are multiple oncogenic drivers and resistance pathways in HR positive breast cancer, and thorough reviews of these complicated mechanisms can be found in the literature elsewhere (26-28). Relevant to this review, 
TABLE 1 | Summary of important phase III clinical trial results for endocrine therapy in metastatic HR positive, HER2 negative breast cancer.

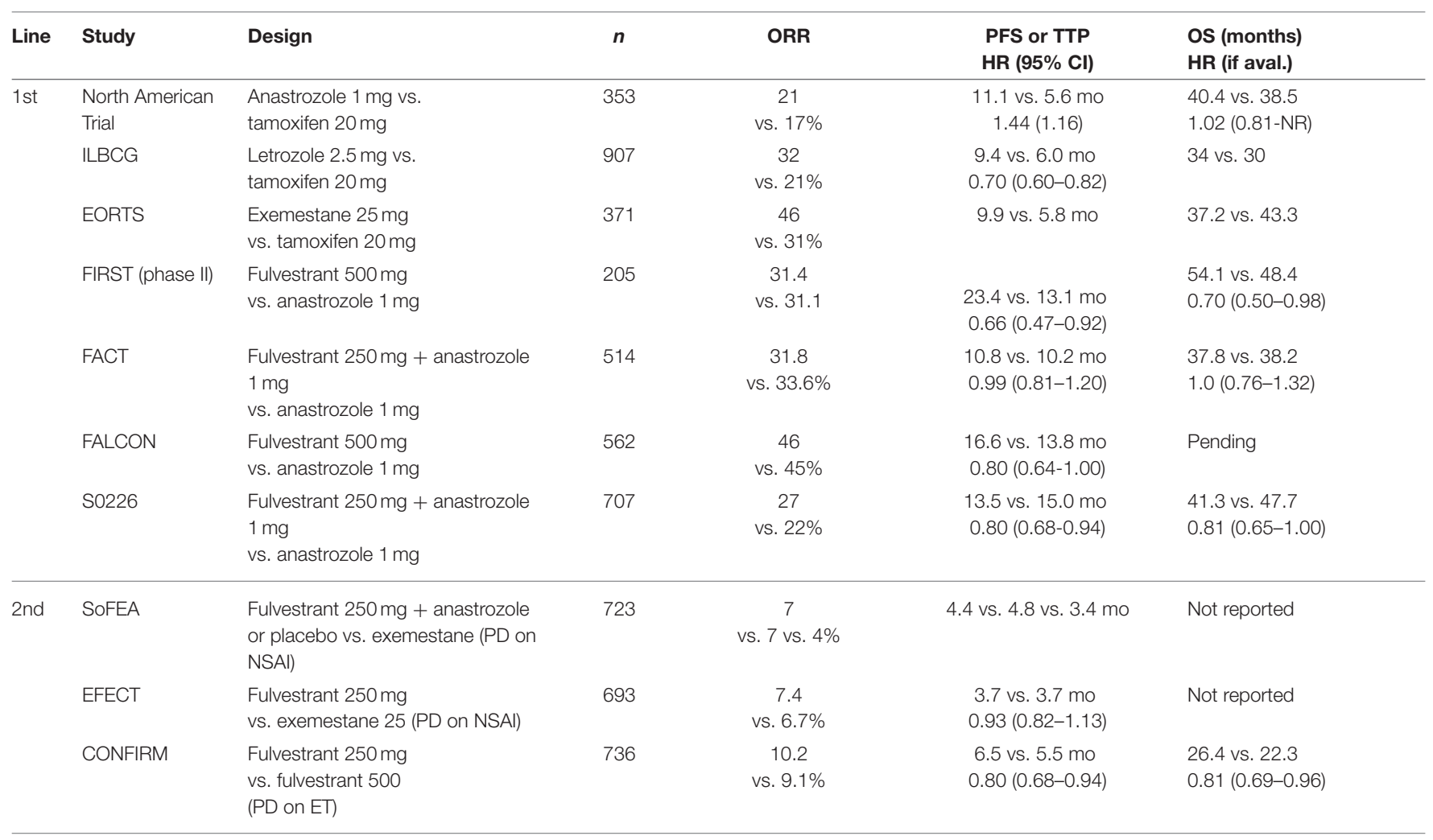

ORR, overall response rate; PFS, progression free survival; TTP, time of progression; HR, hazard ratio; OS, overall survival; NSAl, nonsteroidal aromatase inhibitor; ET, endocrine therapy.

several resistance mechanisms have led to the development of therapeutic strategies, including dysregulation of cell cycle progression, activation of the PI3K/AKT/mammalian target of rapamycin (mTOR) pathway, and ESR1 mutations.

Upregulation of cell cycle regulating molecules, especially those involved in progression of cells from the G1 to $S$ phases, can contribute to endocrine resistance (26). Preclinical work identified CDK 4 as critical for growth of ER positive breast cancer cells resistant to estrogen deprivation (29). Further, in vitro models of fulvestrant resistant breast cancers revealed increased expression of CDK6 when compared to fulvestrant sensitive cells. CDK4/6 is activated when bound to cyclin D1 in the mid-to-late G1 phase of the cell cycle. CDK4/6 phosphorylates $\mathrm{Rb}$ to inactivate its repressive hold on E2F-mediated cell cycle progression $(30,31)$. CDK4/6 activity is particularly relevant in ER positive breast cancers, given that the cyclin D1 gene CCND1 is regulated by ER $\alpha$ (32). Laboratory and clinical studies confirm that CDK4/6 inhibitors potently decrease growth of ER positive breast cancers (33-35). Biomarker analysis of 91 patients with resistance to fulvestrant demonstrated that elevated CDK6 levels associated with a shorter PFS (36).

Molecularly, estrogen activity can induce activation of insulinlike growth factor and the PI3K/AKT and MAPK (mitogen activated protein kinase) pathways, which can downregulate ER and PR cell surface expression (37-41). Increased PI3K/AKT signaling can also result in hormone receptor deactivation, and promote ligand independent growth of malignancies in the absence of endocrine signaling. PI3K/AKT signaling has also been demonstrated to directly phosphorylate the estrogen receptor, resulting in estrogen independent activation of hormone receptors and providing a secondary mechanism of ET resistance (42). PTEN (phosphatase and tensin homolog) is a negative regulator of the PI3K pathway, and PTEN expression is downregulated in the setting of endocrine resistance (43).

Multiple genomic alterations have been identified in MBCs with both intrinsic and acquired resistance, including in ESR1, resulting in constitutive activation of ER. In correlative analysis of several trials, frequency of ESR1 mutations in circulating tumor DNA (ctDNA) is between 25 and 39\% (44-46). ESR1 mutations have been linked to poorer outcomes in patients treated with AI based therapies. In a secondary analysis of the BOLERO-2 trial of exemestane with or without the MTOR inhibitor everolimus, the presence of an ESR1 mutation was associated with reduced OS (47). Data have suggested that SERDs, such as fulvestrant, may overcome ESR mutations. In the SoFEA study, patients the ESR1 mutated tumors had significantly worse outcomes when treated with exemestane, but not when treated with fulvestrant (HR 0.52, $95 \%$ CI $0.30-0.92, p=0.02)(44)$.

Given the effectiveness of ET in the HR positive population, therapies designed to overcome de novo or acquired resistance targeting the aforementioned signaling and cell cycle regulating molecules may be particularly effective at extending OS and PFS in this population. In the next section, we review the small molecule inhibitors that have been approved or are being 
investigated in combination with antiestrogens for the treatment of post-menopausal women with advanced HR positive, HER2 negative breast cancer in the first or later line settings, targeting both intrinsic and acquired resistance.

\section{CDK4/6 Inhibitors}

\section{Palbociclib}

Palbociclib was originally reported in 2009 as PD032991 and noted to selectively inhibit cyclin-dependent kinases 4 and 6 , which resulted in selective inhibition of the proliferation of luminal ER positive breast cancer cell lines, while nonluminal basal cell lines were largely resistant to treatment. Molecular analysis of these cell lines revealed that phosphorylated retinoblastoma $(\mathrm{Rb})$ and cyclin $\mathrm{D} 1$ were elevated while p16 was decreased in sensitive luminal cell lines. This $\mathrm{Rb}$ phosphorylation was inhibited by treatment with palbociclib resulting in G0/G1 cell cycle arrest (48). It is important to note that while the anti-proliferative effects of palbociclib were synergistic with antiestrogen and anti-HER2 therapy in sensitive cell lines (48), in triple negative breast cancer and RB1-proficient cell lines, palbociclib was actually antagonistic to anthracycline mediated cytotoxicity (49). The dose limiting toxicity of palbociclib is neutropenia resulting from cell cycle arrest of marrow hematopoietic stem cells and precursors. This is reversible upon discontinuation of the drug, in contrast to chemotherapeutic agents such as paclitaxel or doxorubicin (50).

PALOMA-1/TRIO-18, a phase II study, assessed the safety and efficacy of palbociclib in conjunction with letrozole as first line treatment of HR positive, HER2 negative advanced breast cancer. The study examined two cohorts, enrolling the first based on receptor status, while the second was enrolled afterwards with additional requirements for amplification of cyclin D1, loss of p16, or both. Both cohorts were randomized (1:1) to receive letrozole $2.5 \mathrm{mg}$ daily with or without oral palbociclib $125 \mathrm{mg}$ daily for 21 days out of a 28-day cycle. The primary endpoint of investigator determined PFS was found to be 10.2 months for letrozole alone, compared to 20.2 months for palbociclib plus letrozole (HR $0.49,95 \%$ CI $0.32-0.75, p=0.0004$ ). In cohort 1 , PFS was 26.1 months for the palbociclib plus letrozole group, vs. 5.7 months for letrozole alone (HR $0.30,95 \%$ CI $0.16-0.57$, $p<0.0001)$. In the second cohort with potential biomarkers, PFS was 18.1 months for the palbociclib plus letrozole group, vs. 11.1 months with letrozole alone (HR 0.51, 95\% CI 0.30$0.85, p=0.0046)$. Accrual to cohort 2 was stopped early due to unplanned interim analysis of cohort 1 and amendment of planned combined cohort analysis. The different outcomes between cohorts 1 and 2 suggested a different behavior of the CCND1 amplified or p16 deficient tumors, and support ER positivity as the most important biomarker for response to AI plus CDK4/6 inhibition, with additional molecular markers of response remaining elusive, as discussed below. In the toxicity analysis, grade 3-4 side effects were more common in the palbociclib group, with neutropenia (54 vs. 1\%) leukopenia (19 vs. $1 \%$ ) and fatigue ( 4 vs. $1 \%$ ) being the most common (33). Based on the results of the PALOMA-1/TRIO 18 study, palbociclib received accelerated approval in combination with letrozole for first line therapy for post-menopausal women with advanced HR positive, HER2 negative breast cancer and subsequent therapy after progression on prior endocrine therapy (51). The follow up phase III study, PALOMA-2, confirmed these results, leading to regular approval of palbociclib (52).

In PALOMA-3, a double-blind phase III study to assess the efficacy and safety of palbociclib plus fulvestrant in ET resistant (define as progression on ET or relapse within 12 months of adjuvant ET) HR positive metastatic breast cancer, the combination of palbociclib plus fulvestrant was associated with significant improvement in PFS compared to fulvestrant and placebo. Median PFS was 9.5 months in the fulvestrant plus palbociclib group, compared to 4.6 months in the fulvestrant plus placebo group (HR $0.46,95 \%$ CI $0.36-0.59, p<0.0001$ ) (53). Analysis of patient reported outcomes revealed that treatment with palbociclib plus fulvestrant resulted in higher global quality of life scores and significant reduction in pain when compared to fulvestrant alone (54).

\section{Ribociclib}

Ribociclib, originally designated LEE011, is an oral small molecule inhibitor of CDK4/6 similar to palbociclib (55). In preclinical models, ribociclib was found to be highly selective for CDK4/6 and function primarily with G1 arrest and regression in some animal models (56). Ribociclib was approved based up on the MONALEESA-2 study, a phase III randomized, doubleblind trial of 668 patients evaluating ribociclib plus letrozole for first line treatment of women with HR positive, HER2 negative MBC. The trial was stopped early after a preplanned interim analysis revealed a significant advantage for the ribociclib containing arm. With a median follow up of 15.3 months, the PFS was significantly longer in the ribociclib containing arm (HR 0.56; 95\% CI, $0.43-0.72 ; p<0.001$ ). Updated analyses show a median PFS of 25.3 months with ribociclib vs. 16.0 months for placebo (57). Common grade $3 / 4$ adverse events in the ribociclib group were neutropenia (59.3 vs. $0.9 \%$ ) and leukopenia (21.0 vs. $0.6 \%)(35)$. MONALEESA-3 is a phase III study of fulvestrant in combination with ribociclib or placebo for both first and second line treatment of HR positive, HER2 negative MBC, with results reported at the 2018 American Society of Clinical Oncology annual meeting. Consistent with other trials, PFS was significantly improved in both the first line (HR $0.577,95 \%$ CI $0.42-0.80$ ) and second line/early relapse settings (HR $0.565,95 \%$ CI 0.43-0.74) (58).

MONALEESA-7 specifically addressed the pre-menopausal group. Presented at the 2017 San Antonio Breast Cancer Symposium (SABCS), this phase III trial compared ribociclib vs. placebo in pre-menopausal woman receiving an AI or tamoxifen with ovarian suppression. Median PFS again favored the ribociclib arm (23.8 vs. 13.0 months, HR $0.55,95 \%$ CI $0.44-0.69, p<0.0001)(59)$.

\section{Abemaciclib}

Abemaciclib is the third agent in the class of CDK4/6 inhibitors that is currently FDA approved.

MONARCH-2, a global, double-blind phase III trial investigating abemaciclib in combination with fulvestrant compared to fulvestrant alone in HR positive, HER2 negative 
MBC with progression on prior ET, demonstrated an improved PFS with the combination therapy (16.4 vs. 9.3 months, HR 0.553 , 95\% CI $0.449-0.681, p<0.001)$. Treatment with abemaciclib was associated with increased rates of diarrhea $(86.4$ vs. $24.7 \%$ ), neutropenia ( 46.0 vs. $4.0 \%$ ), nausea ( 45.1 vs. $22.9 \%$ ), and fatigue (39.9 vs. $26.9 \%)$, as well as an increased rate of thromboembolic events ( 2.0 vs. $0.4 \%$ ) (60). Results of the phase III study of abemaciclib in combination with a non-steroidal AI (MONARCH-3) were recently reported (61). This study allowed the treating physician to determine the non-steroidal AI utilized (79.1\% letrozole, $20.9 \%$ anastrozole). Interim analysis was conducted following 194 PFS events with a median follow up of 17.8 months. Abemaciclib was associated with improved PFS (14.7 months vs. not reached, HR 0.543, 95\% CI 0.41-0.72; $p<0.001)$.

MONARCH-1, a phase II single arm study, evaluated the safety and efficacy of abemaciclib monotherapy in patients with HR positive, HER2 negative metastatic breast cancer of any menopausal status, whose disease had progressed on prior endocrine and chemotherapy. At the time of interim analysis 132 patients had been treated with abemaciclib $200 \mathrm{mg}$ twice daily as monotherapy, with a median of three lines of prior therapy and over $90 \%$ having visceral disease. At 12 months, the ORR was $19.7 \%$, median PFS of 6.0 months, and median OS of 17.7 months (62).

\section{Comparative Efficacy, Sequencing, and Biomarkers}

Palbociclib, ribociclib, and abemaciclib have never been directly compared and have resulted in similar effect sizes (see Table 2). Differences in median PFS are attributable to differing patient populations between studies, with similar hazard ratios in studies across the three approved CDK4/6 inhibitors. After the 2018 ASCO annual meeting, we have results for all of these agents both in the first line setting with AIs and the second line setting in combination with fulvestrant. In addition, MONALESSA-7 and analyses from PALOMA-3 and MONARCH-2 have shown similar efficacy for CDK4/6 inhibitors in combination with ET and ovarian suppression for pre-menopausal women, confirming what many oncologists were already doing in practice $(59,63)$.

While the three agents likely have similar efficacy, the toxicity profiles, dosing schedules, and monitoring required differ (64). Palbociclib and ribociclib, given on a 3 weeks on and 1 week off schedule, both have dose limiting toxicity of neutropenia, while the dose limiting toxicity for daily abemaciclib is diarrhea, perhaps due to its greater affinity for CDK4 over CDK6. Complete blood count (CBC) monitoring is required for all agents. Ribociclib uniquely requires monitoring of liver function tests, electrolytes, and the QT interval. Abemaciclib requires liver function test monitoring as well, and may increase risk of thromboembolic disease. There is no specific benefit of one toxicity profile over another, and patient comorbidities, preferences, and physician familiarity dictate choice between these agents. Packaging provided by drug companies also differs between the agents, with the blister packaging of ribociclib potentially allowing for dose reduction in a more timely manner without the need for a new prescription. Importantly, the financial toxicity of each of these agents can be significant.
While the data for the use of CDK4/6 inhibitors in the first or second line has been impressive, questions remain. Overall survival data from the larger, randomized trials remains to be seen and will likely be difficult to interpret given the long natural history of HR positive MBC. In addition, data clarifying when it is best to sequence a CDK4/6 inhibitor in the course of a patient's therapy options are lacking. Regardless, PFS, quality of life, and delay of chemotherapy are important clinical benefits in the absence of survival data, and it is likely that receiving a CDK4/6 inhibitor at some point is better than never having received one. The decision of use in the first or second line should be based on disease characteristics and patient preference. While the majority of patients will likely receive a CDK4/6 inhibitor in the first line setting, select patients with a long diseasefree interval after adjuvant ET, or small volume or bone-only disease, are likely to have a long PFS interval with ET therapy alone and may opt to delay CDK4/6 inhibitor initiation to a later line.

Despite the promising results, approximately $20 \%$ of patients will not respond to CDK4/6 inhibitors and the majority will develop resistance. Thus far, there has been no subgroup or clinical population that does not derive benefit from CDK4/6 inhibitors, including age, menopausal status, visceral vs. bone metastases, race, or treatment free interval. In addition, an FDA pooled analysis looking at rarer clinical subtypes, including lobular disease, $\mathrm{PR}$ negative, or de novo $\mathrm{MBC}$, found continued benefit to all subtypes (65). It is important to note that the CDK4/6 inhibitors have high and potentially rapid response rates in patients with even advanced visceral disease, and can therefore be used instead of chemotherapy in the absence of true "visceral crisis."

In addition to clinical subtypes, there has been a consistent benefit to CDK4/6 inhibitors across molecular biomarkers thought to be involved in resistance, including $\mathrm{pRb}, \mathrm{p} 16$, Ki67, ESR1 expression, CDKN2A expression, CCND1 expression, PI3KCA mutations, and ESR1 mutations, among others. An abstract presented at the 2018 ASCO annual meeting looking at mRNA expression in MONALESSA2 found a trend toward longer PFS with higher ESR1 expression, and a trend toward more benefit from ribociclib with high ESR1 expression, compared to low (HR 0.39 vs. HR 0.74) (66). Mechanisms of resistance to CDK4/6 inhibitors are reviewed elsewhere and thought to include cyclin E amplification, $\mathrm{Rb}$ loss, CDK2 overexpression, FGFR1 amplification or mutation, and upregulation of the PI3k/AKT pathway $(67,68)$. These investigations have led to the development of clinical trials assessing drug combinations, including CDK4/6 inhibition with PI3K or mTOR inhibitors.

\section{PI3K/AKT/mTOR Inhibitors mTOR Inhibitors}

mTOR is a PI3K kinase with roles in the regulation of PI3K signaling and regulation of protein synthesis and cell growth (69). In breast cancer, activated mTOR signaling is associated with poor patient survival (70) and worse prognosis (71). mTOR can be activated by activating mutations in the PIK3CA gene 
TABLE 2 | Summary of phase III clinical trial results for CDK4/6 inhibitors in metastatic HR positive, HER2 negative breast cancer.

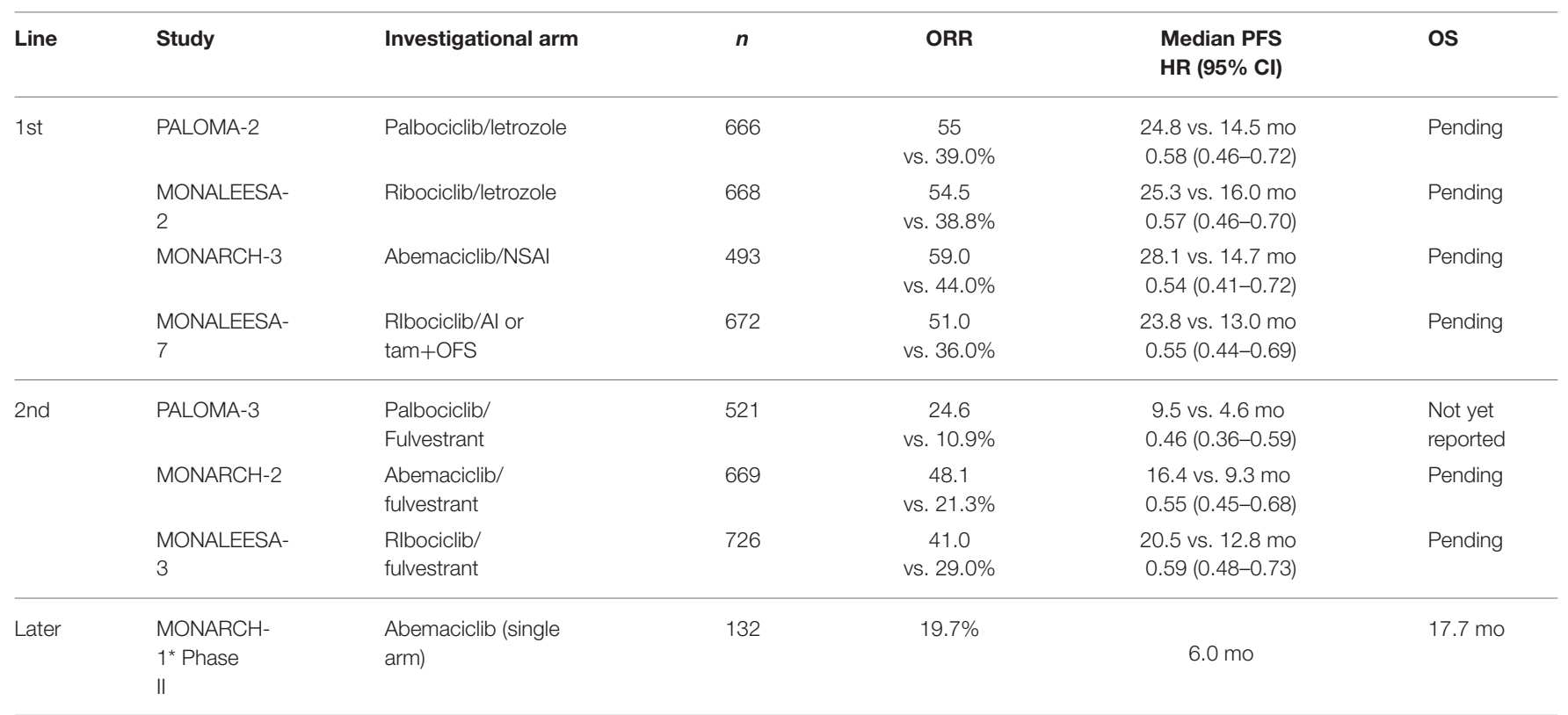

ORR, overall response rate; PFS, progression free survival; HR, hazard ratio; OS, overall survival; NSAl, nonsteroidal aromatase inhibitor.

(72), alteration or mutation of AKT, or loss of PTEN signaling (73). mTOR activation has been associated with resistance to SERMs and AIs $(74,75)$ and inhibition of the mTOR pathway is associated with resensitization of breast cancer cells to hormonal therapy (76). In breast cancer cells resistant to AI, treatment with the MTOR inhibitor everolimus has been associated with dramatically reduced ER expression and increased autophagy in MCF-7 breast cancer cell lines $(77,78)$. Results from phase III trials assessing agents in this pathway are summarized in Table 3.

Based on preclinical and neoadjuvant data, the combination of everolimus plus exemestane was assessed for efficacy and safety in patients with HR positive, HER2 negative breast cancer refractory to non-steroidal AIs in the international double-blind phase III BOLERO-2 study. The primary end point of PFS was met by the study, with the advantage for the combination arm (6.9 vs. 2.8 months, HR 0.43 , 95\% CI $0.35-0.54, p<0.001$ ). Twentythree percent of patients treated with everolimus reported serious adverse events, and $19 \%$ of patients (4\% treated with placebo) discontinued therapy due to adverse events. The most common grade $3 / 4$ toxicities were stomatitis $(8 \%)$, anemia $(6 \%)$, dyspnea (4\%), and pneumonitis (3\%) (79). Further analysis in the BALLET study of everolimus and exemestane in patients previously treated with or without chemotherapy, found a high rate of adverse events, the most common being stomatitis (51.3\%) and confirmed the safety profile was not affected by prior chemotherapy (80). In addition, despite the increased toxicity, there was no evidence of statistically significant improvement in OS (hazard ratio 0.89; 95\% CI 0.73-1.10; $P=0.14)$ (81).

As stomatitis has been a consistent problem in completing everolimus therapy, the phase II SWISH study investigated the use of prophylactic steroid mouthwash. Of the 85 women evaluable for efficacy, only $2 \%$ had grade 2 or worse stomatitis, vs. 33\% historically in BOLERO-2 (82). A similar phase III trial (Oral Care-BC) is investigating the impact of professional dental cleanings by oral surgeons on the incidence of oral mucositis with everolimus therapy. The study is still accruing with estimated primary completion date of March 2019 (NCT02376985) (83).

Vistusertib, an oral dual inhibitor of mTORC1 and mTORC2, was superior to everolimus (an mTORC1 inhibitor) in preclinical models and therefore was investigated in the phase II randomized study MANTA, evaluating fulvestrant alone versus fulvestrant with daily vistusertib, fulvestrant with intermittent vistusertib, and fulvestrant with everolimus. No significant difference was seen between the arms, with the exception of improved PFS in the fulvestrant with everolimus arm compared to fulvestrant with vistusertib (HR 0.64, 95\% CI 0.45-0.91, $p=0.01$ ), and fulvestrant with everolimus compared to fulvestrant alone (HR 0.64, 95\% CI $0.43-0.94, p=0.02)(84)$.

\section{PI3K Inhibitors}

PI3K/AKT signaling plays an important role in the growth and proliferation of tumor cells by acting upstream of the mTOR signaling pathway. This signaling pathway is tightly regulated by the tumor repressor genes PTEN and INPP4B, which are frequently downregulated in breast malignancies, resulting in amplification of the PI3K/AKT/mTOR signal (85). PIK3CA mutations are also frequently mutated in $\mathrm{HR}$ positive breast cancer and are frequently associated with the luminal B subtype of breast cancer (86). Although PIK3CA mutations are frequently detected in breast cancers [32\% in TCGA (87-89)], they have not been associated with clinical outcome (87) or influenced OS (90). However, in multivariate analysis, PIK3CA mutation in conjunction with node positive disease has been associated with unfavorable OS (87). 
TABLE 3 | Summary of phase III clinical trial results for PI3K and mTOR inhibitors in metastatic HR positive, HER2 negative breast cancer.

\begin{tabular}{|c|c|c|c|c|c|c|}
\hline Line & Study & $\begin{array}{l}\text { Investigational } \\
\text { arm }\end{array}$ & $n$ & ORR & $\begin{array}{l}\text { Median PFS } \\
\text { HR }(95 \% \mathrm{CI})\end{array}$ & os \\
\hline \multirow[t]{4}{*}{ 2nd } & BOLERO-2 & $\begin{array}{l}\text { Everolimus/ } \\
\text { exemestane }\end{array}$ & 724 & $\begin{array}{l}9.5 \text { vs. } \\
0.4 \%\end{array}$ & $\begin{array}{l}6.9 \text { vs. } 2.8 \mathrm{mo} \\
0.43(0.35-0.54)\end{array}$ & $\begin{array}{l}31.0 \text { vs. } 26.6 \mathrm{mo} \\
0.89(0.73-1.1)\end{array}$ \\
\hline & BELLE-2 & $\begin{array}{l}\text { Buparlisib/ } \\
\text { fulvestrant }\end{array}$ & 1,147 & $\begin{array}{c}11.8 \\
\text { vs. } 7.7 \%\end{array}$ & $\begin{array}{l}6.9 \text { vs. } 5.0 \mathrm{mo} \\
0.78(0.67-0.89)\end{array}$ & Pending \\
\hline & BELLE-3 & $\begin{array}{l}\text { Buparlisib/ } \\
\text { fulvestrant }\end{array}$ & 432 & $\begin{array}{c}22 \text { vs. } \\
3 \%\end{array}$ & $\begin{array}{l}3.9 \text { vs. } 1.8 \mathrm{mo} \\
0.67(0.53-0.84)\end{array}$ & Pending \\
\hline & SANDPIPER & $\begin{array}{l}\text { Talelisib/ } \\
\text { fulvestrant }\end{array}$ & $\begin{array}{l}516 \text { Pi3K } \\
\text { mutants }\end{array}$ & $\begin{array}{l}28 \text { vs. } \\
11.9 \%\end{array}$ & $\begin{array}{l}7.4 \text { vs. } 5.4 \text { mo } \\
0.70(0.56-0.89)\end{array}$ & Pending \\
\hline
\end{tabular}

ORR, overall response rate; PFS, progression free survival; HR, hazard ratio; OS, overall survival.

There are currently no PI3K inhibitors approved for HR positive breast cancer, although several are in registration trials. The most heavily studied has been buparlisib (BKM120), an oral pan-PI3K inhibitor under examination in the BELLE2 (NCT01610284) and BELLE-3 (NCT01633060) phase III clinical trials in combination with fulvestrant in HR positive, HER2 negative, locally advanced, or MBC refractory to AI or $\mathrm{AI}+\mathrm{mTOR}$ inhibitor, respectively. In BELLE-2, initial PFS data shows a significant advantage for the buparlisib arm (6.9 vs. 5.0 months, HR 0.78, 95\% CI 0.67-0.89, $p<0.001$ ). In BELLE-3, similar results were seen favoring the buparlisib arm, but PFS was short (3.9 vs. 1.8 months, HR $0.67,95 \%$ CI $0.53-0.84$ ), $p<0.001)$. On retrospective analysis of ctDNA, the benefit was restricted to the PIK3CA mutated group, while this was not seen in BELLE-3. In addition, significant side effects were seen, including elevated liver function tests, hyperglycemia, anxiety, and depression, resulting in dose delay and interruption $(91,92)$.

Taselisib (GDC-0032), a selective PI3K inhibitor with greater in vitro activity against mutant isoforms, has been studied in the phase III SANDPIPER trial, with initial results recently reported. Patients with PIK3CA mutated tumors with progression on prior AI were randomized 2:1 to taselisib with fulvestrant or placebo with fulvestrant. An exploratory arm enrolled patients with wild type tumors. In the mutated arms, PFS was improved with the addition of taselisib (7.4 vs. 5.4 months, HR 0.70, $p=0.0037)$. In the exploratory wild type group, there was no difference between the arms, but sample size was small. Fifty percent of patients in the investigational arm developed grade 3 or 4 toxicity, and the majority of patients discontinued treatment due to adverse events, diarrhea being most common (93).

While clinical trials have shown proof of concept for PI3K inhibition in endocrine resistance, the modest efficacy, significant toxicity, and unclear use within the CDK4/6 inhibitor spectrum will likely limit future development of these compounds.

In addition to PI3K inhibitors, several other classes of agents are under investigation to combat endocrine resistance but have yet to provide phase III clinical trial results. Several FGFR inhibitors are under investigation both in combination with endocrine therapy and with other targeted therapies (94). The oral histone deacetylase (HDAC) inhibitor entinostat in combination with exemestane improved DFS and OS over exemestane alone in a phase II trial (95) and the randomized phase III study is ongoing (NCT02115282).

\section{CONCLUSIONS}

HR positive breast cancer is responsible for the majority of breast cancer related deaths. Although outcomes for HR positive breast cancer are excellent in early disease, in the advanced setting, outcomes remain poor with more women dying from HR positive breast cancer than other subtypes combined. In an era of personalized medicine with four small molecule inhibitors already FDA-approved (everolimus, pabociclib, riboclicib, abemaciclib), in addition to others under clinical investigation, the question of how to tailor therapy for patients in this setting is very relevant. Currently there is a lack of definitive biomarkers to identify specific patient populations that will benefit more from one targeted therapy over another.

Without clear biomarkers, selection of ET in the first line metastatic setting depends on clinician/patient preferences, disease burden, and disease biology. While endocrine therapy alone, including aromatase inhibitors, tamoxifen, or fulvestrant, may be appropriate for select patients, the consistent benefits of CDK4/6 inhibitors across multiple subgroups, including those with longer disease free intervals, support their use in the first line for most patients while we await overall survival data. Options for the ET backbone include AIs or fulvestrant for post-menopausal patients, and tamoxifen, AI, or fulvestrant with ovarian suppression for pre-menopausal patients. For most patients, AI (with or without ovarian suppression) and a CDK4/6 inhibitor will likely be most appropriate, with fulvestrant plus CDK4/6 inhibitor used in those who relapsed while on or within 12 months of adjuvant AI therapy, or those with a known ESR1 mutation. Again, individual patient comorbidities, preferences for drug administration, and access should play a role in selection of first line therapy.

In the event of progression, second line treatment should include a CDK4/6 inhibitor if not used in the first line setting. While we await data on the utility of continued CDK $4 / 6$ inhibition or switching agents after progression, options for patients after progression include fulvestrant or an AI alone if not previously used, or exemestane with everolimus. We 
are in favor of enrollment in clinical trials for patients with CDK4/6 inhibitor resistance, including, but not limited to, the MAINTAIN trial evaluating fulvestrant with or without ribociclib after progression on palbociclib or ribociclib (NCT02632045); PACE evaluating fulvestrant, fulvestrant with palbociclib, or fulvestrant with palbociclib plus the anti-PD-1 avelumab in patients progressing on palbociclib (NCT03147287), or TRINITI, evaluating ribociclib with everolimus and exemestane after prior CDK4/6 inhibitor treatment (NCT02732119). For patients progressing rapidly through ET, standard cytotoxic chemotherapy can be tried.

Continued work to evaluate biomarkers of resistance may lead us in the future to a precision medicine approach to therapy after CDK4/6 inhibitor resistance develops. Recent data evaluating ctDNA before and after CD4/6 inhibitor treatment in PALOMA-3 showed the development of Rb, PIK3CA, and ESR1 mutations (96). This study indicated feasibility of detecting genomic changes in the blood, which might guide us to alter therapy in a targeted way. Correlative studies from the ongoing

\section{REFERENCES}

1. Siegel RL, Miller KD, Jemal A. Cancer statistics, 2016. CA Cancer J Clin. (2016) 66:7-30. doi: 10.3322/caac. 21332

2. Reinert T, Barrios $\mathrm{CH}$. Optimal management of hormone receptor positive metastatic breast cancer in 2016. Ther Adv Med Oncol. (2015) 7:304-20. doi: $10.1177 / 1758834015608993$

3. Kaklamani VG, Gradishar WJ. Endocrine therapy in the current management of postmenopausal estrogen receptor-positive metastatic breast cancer. Oncologist (2017) 22:507-17. doi: 10.1634/theoncologist.2015-0464

4. Rugo HS. The breast cancer continuum in hormone-receptor-positive breast cancer in postmenopausal women: evolving management options focusing on aromatase inhibitors. Ann Oncol. (2008) 19:16-27. doi: 10.1093/annonc/mdm282

5. Nagaraj G, Ma C. Revisiting the estrogen receptor pathway and its role in endocrine therapy for postmenopausal women with estrogen receptorpositive metastatic breast cancer. Breast Cancer Res Treat. (2015) 150:231-42. doi: 10.1007/s10549-015-3316-4

6. Curigliano G, Goldhirsch A. To switch or not to switch: implications of sequencing adjuvant endocrine therapy in patients with breast cancer. Nat Clin Pract Oncol. (2007) 4:510-1. doi: 10.1038/ncponc0902

7. Mestres JA, iMolins AB, Martinez LC, Lopez-Muniz JI, Gil EC, de Juan Ferre A, et al. Defining the optimal sequence for the systemic treatment of metastatic breast cancer. Clin Trans Oncol. (2017) 19:149-61. doi: 10.1007/s12094-016-1520-2

8. Cardoso F, Costa A, Senkus E, Aapro M, Andre F, Barrios CH, et al. 3rd ESOESMO International consensus guidelines for advanced breast cancer (ABC 3). Ann Oncol. (2017) 28:3111. doi: 10.1093/annonc/mdx036

9. Verschoor AM, Kuijer A, Verloop J, Van Gils CH, Sonke GS, Jager A, et al. Adjuvant systemic therapy in early breast cancer: impact of guideline changes and clinicopathological factors associated with nonadherence at a nation-wide level. Breast Cancer Res Treat. (2016) 159:357-65. doi: 10.1007/s10549-016-3940-7

10. Klijn JG, Blamey RW, Boccardo F, Tominaga T, Duchateau L, Sylvester R. Combined tamoxifen and luteinizing hormone-releasing hormone (LHRH) agonist versus LHRH agonist alone in premenopausal advanced breast cancer: a meta-analysis of four randomized trials. J Clin Oncol. (2001) 19:343-53. doi: 10.1200/JCO.2001.19.2.343

11. Buzdar AU, Robertson JF, Eiermann W, Nabholtz JM. An overview of the pharmacology and pharmacokinetics of the newer generation aromatase inhibitors anastrozole, letrozole, and exemestane. Cancer (2002) 95:2006-16. doi: $10.1002 /$ cncr.10908 trials mentioned above will likely begin to help us answer these questions.

Single agent endocrine therapy and combinations with targeted therapy offer a range of options for patients with metastatic HR positive breast cancer. Currently, the optimal sequencing of these therapies is unclear, and should be based on patient characteristics, clinical judgement, and disease biology. While sequential lines of ET combinations can improve outcomes for patients, many acquire endocrine resistance. Ongoing research to identify biomarkers and optimal sequencing for individual patients is awaited, with hopes to continue to improve outcomes and quality of life for patients with HR positive metastatic breast cancer.

\section{AUTHOR CONTRIBUTIONS}

TB and JM are responsible for the content of this article. TB and $\mathrm{VJ}$ are responsible for the intellectual content, analysis, and revision of the manuscript.
12. Miller WR, Bartlett J, Brodie AM, Brueggemeier RW, di Salle E, Lonning PE, et al. Aromatase inhibitors: are there differences between steroidal and nonsteroidal aromatase inhibitors and do they matter? Oncologist (2008) 13:829-37. doi: 10.1634/theoncologist.2008-0055

13. Ferretti G, Bria E, Giannarelli D, Felici A, Papaldo P, Fabi A, et al. Secondand third-generation aromatase inhibitors as first-line endocrine therapy in postmenopausal metastatic breast cancer patients: a pooled analysis of the randomised trials. Br J Cancer (2006) 94:1789-96. doi: 10.1038/sj.bjc.6603194

14. Bonneterre J, Thurlimann B, Robertson JF, Krzakowski M, Mauriac L, Koralewski P, et al. Anastrozole versus tamoxifen as first-line therapy for advanced breast cancer in 668 postmenopausal women: results of the Tamoxifen or Arimidex Randomized Group Efficacy and Tolerability study. J Clin Oncol. (2000) 18:3748-57. doi: 10.1200/JCO.2000.18.22.3748

15. Di Leo A, Jerusalem G, Petruzelka L, Torres R, Bondarenko IN, Khasanov $\mathrm{R}$, et al. Results of the CONFIRM phase III trial comparing fulvestrant $250 \mathrm{mg}$ with fulvestrant $500 \mathrm{mg}$ in postmenopausal women with estrogen receptor-positive advanced breast cancer. J Clin Oncol. (2010) 28:4594-600. doi: 10.1200/JCO.2010.28.8415

16. Di Leo A, Jerusalem G, Petruzelka L, Torres R, Bondarenko IN, Khasanov $\mathrm{R}$, et al. Final overall survival: fulvestrant $500 \mathrm{mg}$ vs $250 \mathrm{mg}$ in the randomized CONFIRM trial. J Natl Cancer Instit. (2014) 106:djt337. doi: $10.1093 /$ jnci/djt337

17. Robertson JF, Lindemann J, Garnett S, Anderson E, Nicholson RI, Kuter I, et al. A good drug made better: the fulvestrant dose-response story. Clin Breast Cancer (2014) 14:381-9. doi: 10.1016/j.clbc.2014.06.005

18. Kuter I, Gee JM, Hegg R, Singer CF, Badwe RA, Lowe ES, et al. Dosedependent change in biomarkers during neoadjuvant endocrine therapy with fulvestrant: results from NEWEST, a randomized Phase II study. Breast Cancer Res Treat. (2012) 133:237-46. doi: 10.1007/s10549-011-1947-7

19. Robertson JF, Lindemann JP, Llombart-Cussac A, Rolski J, Feltl D, Dewar J, et al. Fulvestrant $500 \mathrm{mg}$ versus anastrozole $1 \mathrm{mg}$ for the firstline treatment of advanced breast cancer: follow-up analysis from the randomized 'FIRST' study. Breast Cancer Res Treat. (2012) 136:503-11. doi: $10.1007 /$ s10549-012-2192-4

20. Robertson JFR, Bondarenko IM, Trishkina E, Dvorkin M, Panasci L, Manikhas A, et al. Fulvestrant $500 \mathrm{mg}$ versus anastrozole $1 \mathrm{mg}$ for hormone receptor-positive advanced breast cancer (FALCON): an international, randomised, double-blind, phase 3 trial. Lancet (2016) 388:2997-3005. doi: 10.1016/S0140-6736(16)32389-3

21. Chia S, Gradishar W, Mauriac L, Bines J, Amant F, Federico M, et al. Double-blind, randomized placebo controlled trial of fulvestrant compared with exemestane after prior nonsteroidal aromatase inhibitor therapy 
in postmenopausal women with hormone receptor-positive, advanced breast cancer: results from EFECT. J Clin Oncol. (2008) 26:1664-70. doi: 10.1200/JCO.2007.13.5822

22. Bergh J, Jonsson PE, Lidbrink EK, Trudeau M, Eiermann W, Brattstrom D, et al. FACT: an open-label randomized phase III study of fulvestrant and anastrozole in combination compared with anastrozole alone as first-line therapy for patients with receptor-positive postmenopausal breast cancer. $J$ Clin Oncol. (2012) 30:1919-25. doi: 10.1200/JCO.2011.38.1095

23. Mehta RS, Barlow WE, Albain KS, Vandenberg TA, Dakhil SR, Tirumali NR, et al. Combination anastrozole and fulvestrant in metastatic breast cancer. $N$ Engl J Med. (2012) 367:435-44. doi: 10.1056/NEJMoa1201622

24. Johnston SR, Kilburn LS, Ellis P, Dodwell D, Cameron D, Hayward L, et al. Fulvestrant plus anastrozole or placebo versus exemestane alone after progression on non-steroidal aromatase inhibitors in postmenopausal patients with hormone-receptor-positive locally advanced or metastatic breast cancer (SoFEA): a composite, multicentre, phase 3 randomised trial. Lancet Oncol. (2013) 14:989-98. doi: 10.1016/S1470-2045(13)70322-X

25. Lee CI, Goodwin A, Wilcken N. Fulvestrant for hormone-sensitive metastatic breast cancer. Cochr Database Syst Rev. (2017) 1:CD011093. doi: 10.1002/14651858.CD011093

26. Osborne CK, Schiff R. Mechanisms of endocrine resistance in breast cancer. Annu Rev Med. (2011) 62:233-47. doi: 10.1146/annurev-med-070909-182917

27. Musgrove EA, Sutherland RL. Biological determinants of endocrine resistance in breast cancer. Nat Rev Cancer (2009) 9:631-43. doi: 10.1038/ nrc2713

28. Murphy CG, Dickler MN. Endocrine resistance in hormone-responsive breast cancer: mechanisms and therapeutic strategies. Endocr Relat Cancer (2016) 23:R337-52. doi: 10.1530/ERC-16-0121

29. Miller TW, Balko JM, Fox EM, Ghazoui Z, Dunbier A, Anderson H, et al. ERalpha-dependent E2F transcription can mediate resistance to estrogen deprivation in human breast cancer. Cancer Disc. (2011) 1:338-51. doi: 10.1158/2159-8290.CD-11-0101

30. Sherr CJ, Roberts JM. CDK inhibitors: positive and negative regulators of G1phase progression. Genes Dev. (1999) 13:1501-12. doi: 10.1101/gad.13.12.1501

31. Weinberg RA. The retinoblastoma protein and cell cycle control. Cell (1995) 81:323-30. doi: 10.1016/0092-8674(95)90385-2

32. Planas-Silva MD, Donaher JL, Weinberg RA. Functional activity of ectopically expressed estrogen receptor is not sufficient for estrogen-mediated cyclin D1 expression. Cancer Res. (1999) 59:4788-92.

33. Finn RS, Crown JP, Lang I, Boer K, Bondarenko IM, Kulyk SO, et al. The cyclin-dependent kinase $4 / 6$ inhibitor palbociclib in combination with letrozole versus letrozole alone as first-line treatment of oestrogen receptor-positive, HER2-negative, advanced breast cancer (PALOMA1/TRIO-18): a randomised phase 2 study. Lancet Oncol. (2015) 16:25-35. doi: 10.1016/S1470-2045(14)71159-3

34. Turner NC, Huang Bartlett C, Cristofanilli M. Palbociclib in hormonereceptor-positive advanced breast cancer. N Engl J Med. (2015) 373:1672-3. doi: 10.1056/NEJMc1510345

35. Hortobagyi GN, Stemmer SM, Burris HA, Yap YS, Sonke GS, Paluch-Shimon $\mathrm{S}$, et al. Ribociclib as first-line therapy for HR-positive, advanced breast cancer. N Engl J Med. (2016) 375:1738-48. doi: 10.1056/NEJMoa1609709

36. Alves CL, Elias D, Lyng M, Bak M, Kirkegaard T, Lykkesfeldt AE, et al. High CDK6 protects cells from fulvestrant-mediated apoptosis and is a predictor of resistance to fulvestrant in estrogen receptor-positive metastatic breast cancer. Clin Cancer Res. (2016) 22:5514-26. doi: 10.1158/1078-0432.CCR-15-1984

37. Bayliss J, Hilger A, Vishnu P, Diehl K, El-Ashry D. Reversal of the estrogen receptor negative phenotype in breast cancer and restoration of antiestrogen response. Clin Cancer Res. (2007) 13:7029-36. doi: 10.1158/1078-0432.CCR-07-0587

38. Lopez-Tarruella S, Schiff R. The dynamics of estrogen receptor status in breast cancer: re-shaping the paradigm. Clin Cancer Res. (2007) 13:6921-5. doi: 10.1158/1078-0432.CCR-07-1399

39. Cui X, Zhang P, Deng W, Oesterreich S, Lu Y, Mills GB, et al. Insulinlike growth factor-I inhibits progesterone receptor expression in breast cancer cells via the phosphatidylinositol 3-kinase/Akt/mammalian target of rapamycin pathway: progesterone receptor as a potential indicator of growth factor activity in breast cancer. Mol Endocrinol. (2003) 17:575-88. doi: $10.1210 /$ me.2002-0318
40. Cui X, Schiff R, Arpino G, Osborne CK, Lee AV. Biology of progesterone receptor loss in breast cancer and its implications for endocrine therapy. J Clin Oncol. (2005) 23:7721-35. doi: 10.1200/JCO.2005.09.004

41. Creighton CJ, Fu X, Hennessy BT, Casa AJ, Zhang Y, Gonzalez-Angulo AM, et al. Proteomic and transcriptomic profiling reveals a link between the PI3K pathway and lower estrogen-receptor (ER) levels and activity in ER+ breast cancer. Breast Cancer Res. (2010) 12:R40. doi: 10.1186/bcr2594

42. Campbell RA, Bhat-Nakshatri P, Patel NM, Constantinidou D, Ali S, Nakshatri H. Phosphatidylinositol 3-kinase/AKT-mediated activation of estrogen receptor alpha: a new model for anti-estrogen resistance. J Biol Chem. (2001) 276:9817-24. doi: 10.1074/jbc.M010840200

43. Fu X, Creighton CJ, Biswal NC, Kumar V, Shea M, Herrera S, et al. Overcoming endocrine resistance due to reduced PTEN levels in estrogen receptor-positive breast cancer by co-targeting mammalian target of rapamycin, protein kinase $\mathrm{B}$, or mitogen-activated protein kinase kinase. Breast Cancer Res. (2014) 16:430. doi: 10.1186/s13058-014-0430-x

44. Fribbens C, O’Leary B, Kilburn L, Hrebien S, Garcia-Murillas I, Beaney $\mathrm{M}$, et al. Plasma ESR1 mutations and the treatment of estrogen receptor-positive advanced breast cancer. J Clin Oncol. (2016) 34:2961-8. doi: 10.1200/JCO.2016.67.3061

45. Turner NC, Jiang Y, O'Leary C, Hrebien S, Massimo C, Andre F, et al. Efficacy of palbociclib plus fulvestrant $(\mathrm{P}+\mathrm{F})$ in patients (pts) with metastatic breast cancer (MBC) and ESR1 mutations (mus) in circulating tumor DNA (ctDNA). J Clin Oncol. (2016) 34(15 Suppl.):512. doi: 10.1200/JCO.2016.34.15

46. Spoerke JM, Gendreau S, Walter K, Qiu J, Wilson TR, Savage H, et al. Heterogeneity and clinical significance of ESR1 mutations in ER-positive metastatic breast cancer patients receiving fulvestrant. Nat Commun. (2016) 7:11579. doi: 10.1038/ncomms11579

47. Chandarlapaty S, Chen D, He W, Sung P, Samoila A, You D, et al. Prevalence of ESR1 mutations in cell-free DNA and outcomes in metastatic breast cancer: a secondary analysis of the BOLERO-2 clinical trial. JAMA Oncol. (2016) 2:1310-5. doi: 10.1001/jamaoncol.2016.1279

48. Finn RS, Dering J, Conklin D, Kalous O, Cohen DJ, Desai AJ, et al. PD 0332991, a selective cyclin D kinase 4/6 inhibitor, preferentially inhibits proliferation of luminal estrogen receptor-positive human breast cancer cell lines in vitro. Breast Cancer Res. (2009) 11:R77. doi: 10.1186/bcr2419

49. McClendon AK, Dean JL, Rivadeneira DB, Yu JE, Reed CA, Gao E, et al. CDK4/6 inhibition antagonizes the cytotoxic response to anthracycline therapy. Cell Cycle (2012) 11:2747-55. doi: 10.4161/cc.21127

50. Hu W, Sung T, Jessen BA, Thibault S, Finkelstein MB, Khan NK, et al. Mechanistic investigation of bone marrow suppression associated with Palbociclib and its differentiation from cytotoxic chemotherapies. Clin Cancer Res. (2016) 22:2000-8. doi: 10.1158/1078-0432.CCR-15-1421

51. Lu J. Palbociclib: a first-in-class CDK4/CDK6 inhibitor for the treatment of hormone-receptor positive advanced breast cancer. J Hematol Oncol. (2015) 8:98. doi: 10.1186/s13045-015-0194-5

52. Finn RS, Martin M, Rugo HS, Jones S, Im SA, Gelmon K, et al. Palbociclib and letrozole in advanced breast cancer. N Engl J Med. (2016) 375:1925-36. doi: 10.1056/NEJMoa1607303

53. Cristofanilli M, Turner NC, Bondarenko I, Ro J, Im SA, Masuda N, et al. Fulvestrant plus palbociclib versus fulvestrant plus placebo for treatment of hormone-receptor-positive, HER2-negative metastatic breast cancer that progressed on previous endocrine therapy (PALOMA-3): final analysis of the multicentre, double-blind, phase 3 randomised controlled trial. Lancet Oncol. (2016) 17:425-39. doi: 10.1016/S1470-2045(15)00613-0

54. Harbeck N, Iyer S, Turner N, Cristofanilli M, Ro J, Andre F, et al. Quality of life with palbociclib plus fulvestrant in previously treated hormone receptor-positive, HER2-negative metastatic breast cancer: patient-reported outcomes from the PALOMA-3 trial. Ann Oncol. (2016) 27:1047-54. doi: 10.1093/annonc/mdw139

55. Syed YY. Ribociclib: first global approval. Drugs (2017) 77:799-807. doi: 10.1007/s40265-017-0742-0

56. Kim S, Loo A, Chopra R, Caponigro G, Huang A, Vora S, et al. Abstract PR02: LEE011: an orally bioavailable, selective small molecule inhibitor of CDK4/6Reactivating Rb in cancer. Mol Cancer Ther. (2013) 12(11 Suppl.):PR02. doi: 10.1158/1535-7163.TARG-13-PR02

57. Hortobagyi GN, Stemmer SM, Burris HA, Yap YS, Sonke GS, PaluchShimon S, et al. Updated results from MONALEESA-2, a phase 3 trial of 
first-line ribociclib + letrozole in hormone receptor-positive (HR+), HER2negative (HER2-), advanced breast cancer (ABC). J Clin Oncol. (2017) 35(15 Suppl.):1038. doi: 10.1093/annonc/mdy155

58. Slamon DJ, Neven P, Chia S, Fasching PA, De Laurentiis M, Im SA, et al. Phase III randomized study of Ribociclib and Fulvestrant in hormone receptorpositive, human epidermal growth factor receptor 2-negative advanced breast cancer: MONALEESA-3. J Clin Oncol. (2018) 2018:JCO2018789909. doi: 10.1200/JCO.2018.78.9909

59. Tripathy D, Im SA, Colleoni M, Franke F, Bardia A, Harbeck N, et al. Ribociclib plus endocrine therapy for premenopausal women with hormone-receptor-positive, advanced breast cancer (MONALEESA7): a randomised phase 3 trial. Lancet Oncol. (2018) 19:904-15. doi: 10.1016/S1470-2045(18)30292-4

60. Sledge GW Jr, Toi M, Neven P, Sohn J, Inoue K, Pivot X, et al. MONARCH 2: Abemaciclib in combination with fulvestrant in women with HR+/HER2advanced breast cancer who had progressed while receiving endocrine therapy. J Clin Oncol. (2017) 35:2875-84. doi: 10.1200/JCO.2017.73.7585

61. Goetz MP, Toi M, Campone M, Sohn J, Paluch-Shimon S, Huober J, et al. Monarch 3: Abemaciclib as initial therapy for advanced breast cancer. J Clin Oncol. (2017) 35, 3638-46. doi: 10.1200/JCO.2017.75.6155

62. Dickler MN, Tolaney SM, Rugo HS, Cortes J, Dieras V, Patt D, et al. MONARCH 1, a phase II study of Abemaciclib, a CDK4 and CDK6 inhibitor, as a single agent, in patients with refractory HR+/HER2- metastatic breast cancer. Clin Cancer Res. (2017) 23:5218-24. doi: 10.1158/1078-0432.CCR-17-0754

63. Loibl S, Turner NC, Ro J, Cristofanilli M, Iwata H, Im SA, et al. Palbociclib combined with fulvestrant in premenopausal women with advanced breast cancer and prior progression on endocrine therapy: PALOMA-3 results. Oncologist (2017) 22:1028-38. doi: 10.1634/theoncologist.2017-0072

64. Abraham J, Coleman R, Elias A, Holmes FA, Kalinsky K, Kittaneh M, et al. Use of cyclin-dependent kinase (CDK) 4/6 inhibitors for hormone receptor-positive, human epidermal growth factor receptor 2-negative, metastatic breast cancer: a roundtable discussion by The Breast Cancer Therapy Expert Group (BCTEG). Breast Cancer Res Treat. (2018) 171:11-20. doi: 10.1007/s10549-018-4783-1

65. Gao JJ, Cheng J, Bloomquist E, Schroeder R, Amiri-Kordestani L, Sridhara R, et al. Benefit of CDK 4/6 inhibition in less common breast cancer subsets: a U.S. Food and Drug Administration pooled analysis. J Clin Oncol. (2018) 36(Suppl. 1024). doi: 10.1200/JCO.2018.36.15_suppl.1024

66. Hortobagyi GN, Paluch-Shimon S, Pekrakova K, Villanueva C, Chan A, Nusch A, et al. First-line ribociclib (RIB) + letrozole (LET) in hormone receptorpositive (HR+), HER2-negative (HER2-) advanced breast cancer (ABC): MONALEESA-2 biomarker analyses. J Clin Oncol. (2018) 36(Suppl. 1022). doi: 10.1200/JCO.2018.36.15_suppl.1022

67. Yang C, Li Z, Bhatt T, Dickler M, Giri D, Scaltriti M, et al. Acquired CDK6 amplification promotes breast cancer resistance to CDK4/6 inhibitors and loss of ER signaling and dependence. Oncogene (2017) 36:2255-64. doi: 10.1038/onc.2016.379

68. Guarducci C, Bonechi M, Boccalini G, Benelli M, Risi E, Di Leo A, et al. Mechanisms of resistance to CDK4/6 inhibitors in breast cancer and potential biomarkers of response. Breast Care (2017) 12:304-8. doi: 10.1159/000484167

69. Zarogoulidis P, Lampaki S, Turner JF, Huang H, Kakolyris S, Syrigos K, et al. mTOR pathway: a current, up-to-date mini-review (Review). Oncol Lett. (2014) 8:2367-70. doi: 10.3892/ol.2014.2608

70. Xu K, Liu P, Wei W. mTOR signaling in tumorigenesis. Biochim Biophys Acta (2014) 1846:638-54. doi: 10.1016/j.bbcan.2014.10.007

71. Wazir U, Newbold RF, Jiang WG, Sharma AK, Mokbel K. Prognostic and therapeutic implications of mTORC1 and Rictor expression in human breast cancer. Oncol Rep. (2013) 29:1969-74. doi: 10.3892/or.2013.2346

72. McCubrey JA, Steelman LS, Chappell WH, Abrams SL, Montalto G, Cervello M, et al. Mutations and deregulation of Ras/Raf/MEK/ERK and $\mathrm{PI} 3 \mathrm{~K} / \mathrm{PTEN} / \mathrm{Akt} / \mathrm{mTOR}$ cascades which alter therapy response. Oncotarget (2012) 3:954-87. doi: 10.18632/oncotarget.652

73. Strimpakos AS, Karapanagiotou EM, Saif MW, Syrigos KN. The role of mTOR in the management of solid tumors: an overview. Cancer Treat Rev. (2009) 35:148-59. doi: 10.1016/j.ctrv.2008.09.006

74. Viedma-Rodriguez R, Baiza-Gutman L, Salamanca-Gomez F, Diaz-Zaragoza M, Martinez-Hernandez G, Ruiz Esparza-Garrido R, et al. Mechanisms associated with resistance to tamoxifen in estrogen receptor-positive breast cancer (review). Oncol Rep. (2014) 32:3-15. doi: 10.3892/or.2014.3190

75. Miller TW, Hennessy BT, Gonzalez-Angulo AM, Fox EM, Mills GB, Chen H, et al. Hyperactivation of phosphatidylinositol-3 kinase promotes escape from hormone dependence in estrogen receptor-positive human breast cancer. $J$ Clin Invest. (2010) 120:2406-13. doi: 10.1172/JCI41680

76. deGraffenried LA, Friedrichs WE, Russell DH, Donzis EJ, Middleton AK, Silva $\mathrm{JM}$, et al. Inhibition of mTOR activity restores tamoxifen response in breast cancer cells with aberrant Akt Activity. Clin Cancer Res. (2004) 10:8059-67. doi: 10.1158/1078-0432.CCR-04-0035

77. Lui A, New J, Ogony J, Thomas S, Lewis-Wambi J. Everolimus downregulates estrogen receptor and induces autophagy in aromatase inhibitor-resistant breast cancer cells. BMC Cancer (2016) 16:487. doi: 10.1186/s12885-016-2490-z

78. Gelsomino L, Gu G, Rechoum Y, Beyer AR, Pejerrey SM, Tsimelzon A, et al. ESR1 mutations affect anti-proliferative responses to tamoxifen through enhanced cross-talk with IGF signaling. Breast Cancer Res Treat. (2016) 157:253-65. doi: 10.1007/s10549-016-3829-5

79. Baselga J, Campone M, Piccart M, Burris HA III, Rugo HS, Sahmoud T, et al. Everolimus in postmenopausal hormone-receptor-positive advanced breast cancer. N Engl J Med. (2012) 366:520-9. doi: 10.1056/NEJMoa1109653

80. Jerusalem G, Mariani G, Ciruelos EM, Martin M, Tjan-Heijnen VC, Neven $\mathrm{P}$, et al. Safety of everolimus plus exemestane in patients with hormonereceptor-positive, HER2-negative locally advanced or metastatic breast cancer progressing on prior non-steroidal aromatase inhibitors: primary results of a phase IIIb, open-label, single-arm, expanded-access multicenter trial (BALLET). Ann Oncol. (2016) 27:1719-25. doi: 10.1093/annonc/mdw249

81. Piccart M, Hortobagyi GN, Campone M, Pritchard KI, Lebrun F, Ito Y, et al. Everolimus plus exemestane for hormone-receptor-positive, human epidermal growth factor receptor-2-negative advanced breast cancer: overall survival results from BOLERO-2dagger. Ann Oncol. (2014) 25:2357-62. doi: $10.1093 /$ annonc/mdu456

82. Rugo HS, Seneviratne L, Beck JT, Glaspy JA, Peguero JA, Pluard T), et al. Prevention of everolimus-related stomatitis in women with hormone receptor-positive, HER2-negative metastatic breast cancer using dexamethasone mouthwash (SWISH): a single-arm, phase 2 trial. Lancet Oncol. (2017). 18:654-62. doi: 10.1016/S1470-2045(17)30109-2

83. Niikura N, Ota Y, Hayashi N, Naito M, Kashiwabara K, Watanabe K, et al. Evaluation of oral care to prevent oral mucositis in estrogen receptor-positive metastatic breast cancer patients treated with everolimus (Oral Care-BC): randomized controlled phase III trial. Jap J Clin Oncol. (2016) 46:879-82. doi: $10.1093 /$ jjco/hyw077

84. Schmid P, Zaiss M, Harper-Wynne C, Ferreira M, Dubey S, Chan S, et al. MANTA - a randomized phase II study of fulvestrant in combination with the dual mTOR inhibitor AZD2014 or everolimus or fulvestrant alone in estrogen receptor-positive advanced or metastatic breast cancer. In: Oral Presentation At: San Antonio Breast Cancer Symposium 2017. San Antonio, TX (2017).

85. Dey N, De P, Leyland-Jones B. PI3K-AKT-mTOR inhibitors in breast cancers: from tumor cell signaling to clinical trials. Pharmacol Ther. (2017) 175:91106. doi: 10.1016/j.pharmthera.2017.02.037

86. Natrajan R, Lambros MB, Geyer FC, Marchio C, Tan DS, Vatcheva R, et al. Loss of $16 \mathrm{q}$ in high grade breast cancer is associated with estrogen receptor status: evidence for progression in tumors with a luminal phenotype? Genes Chromosomes Cancer (2009) 48:351-65. doi: 10.1002/gcc.20646

87. Cuorvo LV, Verderio P, Ciniselli CM, Girlando S, Decarli N, Leonardi E, et al. PI3KCA mutation status is of limited prognostic relevance in ER-positive breast cancer patients treated with hormone therapy. Virchows Archiv (2014) 464:85-93. doi: 10.1007/s00428-013-1500-7

88. Cerami E, Gao J, Dogrusoz U, Gross BE, Sumer SO, Aksoy BA, et al. The cBio cancer genomics portal: an open platform for exploring multidimensional cancer genomics data. Cancer Disc. (2012) 2:401-4. doi: 10.1158/2159-8290.CD-12-0095

89. Gao J, Aksoy BA, Dogrusoz U, Dresdner G, Gross B, Sumer SO, et al. Integrative analysis of complex cancer genomics and clinical profiles using the cBioPortal. Sci Signal. (2013) 6:11. doi: 10.1126/scisignal.2004088

90. Adamczyk A, Niemiec J, Janecka A, Harazin-Lechowska A, Ambicka A, GrelaWojewoda A, et al. Prognostic value of PIK3CA mutation status, PTEN and androgen receptor expression for metastasis-free survival in HER2-positive 
breast cancer patients treated with trastuzumab in adjuvant setting. Polish J Pathol. (2015) 66:133-41. doi: 10.5114/pjp.2015.53009

91. Baselga J, Im SA, Iwata H, Cortes J, De Laurentiis M, Jiang Z, et al. Buparlisib plus fulvestrant versus placebo plus fulvestrant in postmenopausal, hormone receptor-positive, HER2-negative, advanced breast cancer (BELLE2): a randomised, double-blind, placebo-controlled, phase 3 trial. Lancet Oncol. (2017) 18:904-16. doi: 10.1016/S1470-2045(17)30376-5

92. Di Leo A, Johnston S, Lee KS, Ciruelos E, Lonning PE, Janni W, et al. Buparlisib plus fulvestrant in postmenopausal women with hormonereceptor-positive, HER2-negative, advanced breast cancer progressing on or after mTOR inhibition (BELLE-3): a randomised, double-blind, placebo-controlled, phase 3 trial. Lancet Oncol. (2018) 19:87-100. doi: 10.1016/S1470-2045(17)30688-5

93. Baselga J, Dent SF, Cortés J, Im Y-H, Diéras V, Harbeck N, et al. Phase III study of taselisib (GDC-0032) + fulvestrant (FULV) v FULV in patients (pts) with estrogen receptor (ER)-positive, PIK3CA-mutant (MUT), locally advanced or metastatic breast cancer (MBC): primary analysis from SANDPIPER. 2018 ASCO Annual Meeting. J Clin Oncol. (2018) 36(Suppl LBA1006).

94. Perez-Garcia J, Munoz-Couselo E, Soberino J, Racca F, Cortes J. Targeting FGFR pathway in breast cancer. Breast (2018) 37:126-33. doi: 10.1016/j.breast.2017.10.014
95. Yardley DA, Ismail-Khan RR, Melichar B, Lichinitser M, Munster PN, Klein PM, et al. Randomized phase II, double-blind, placebo-controlled study of exemestane with or without entinostat in postmenopausal women with locally recurrent or metastatic estrogen receptor-positive breast cancer progressing on treatment with a nonsteroidal aromatase inhibitor. J Clin Oncol. (2013) 31:2128-35. doi: 10.1200/JCO.2012.43.7251

96. Turner NC, O'Leary B, Cutts R, Liu Y, Hrebien S, Huang X, et al. Genetic landscape of resistance to CDK4/6 inhibition in circulating tumor DNA (ctDNA) analysis of the PALOMA3 trial of palbociclib and fulvestrant versus placebo and fulvestrant. J Clin Oncol. (2018) 36(Suppl. 1001).

Conflict of Interest Statement: The authors declare that the research was conducted in the absence of any commercial or financial relationships that could be construed as a potential conflict of interest.

Copyright (c) 2018 Ballinger, Meier and Jansen. This is an open-access article distributed under the terms of the Creative Commons Attribution License (CC BY). The use, distribution or reproduction in other forums is permitted, provided the original author(s) and the copyright owner(s) are credited and that the original publication in this journal is cited, in accordance with accepted academic practice. No use, distribution or reproduction is permitted which does not comply with these terms. 